\title{
Nonlinear optical response of a 2D quantum dot supercrystal
}

\author{
Ramil Malikov ${ }^{1, *}$, Igor Ryzhov², and Victor Malyshev ${ }^{3}$ \\ ${ }^{1}$ M. Akmullah State Pedagogical University of Bashkortostan, 450000 Ufa, Russia \\ ${ }^{2}$ A.I. Hertsen State Pedagogical University of Russia, 191186 St.-Petersburg, Russia \\ ${ }^{3}$ Institute for Advanced Materials, University of Groningen, 9747 AG Groningen, The Netherlands
}

\begin{abstract}
We investigate theoretically the nonlinear optical response of a two-dimensional supercrystal comprized of semiconductor quantum dots. An isolated quantum dot is modeled as a three-level ladder-like system with ground, one-exciton, and biexciton states. It is shown that the optical response of supercrystal demonstrate a rich nonlinear dynamics, including bistability, self-oscillations, and dynamical chaos.
\end{abstract}

Supercrystals (SCs) comprising semiconductor quantum dots (SQDs) represent a class of new materials not existing in nature. Modern nanotechnology has in its disposal a variety of methods to design such systems [1]. Optical properties of SCs depend on the SQD's size, shape, and chemical composition, as well as on the lattice geometry and can be easily controlled [2].

We conduct a theoretical study of the optical response of a two-dimensional SC. Due to a high density of SQDs in the SC, the SQD-SQD dipole-dipole interaction plays an important role in the SC's optical response, both linear and nonlinear. This interaction provides a positive feedback which, together with the SQD's nonlinearity, results in a rich optical dynamics of SC, including bistability, self-oscillations, and dynamical chaos.

It is assumed that the SC undergoes an action of an external field of an amplitude $\mathrm{E}_{0}$ and frequency $\omega_{0}$, which is quasiresonant with the SQD allowed transitions. A single SQD is modelled as a three-level ladder-like quantum system with the ground $|1\rangle$, one-exciton $|2\rangle$, and bi-exction $|3\rangle$ states. Only the optical transitions $|1\rangle \leftrightarrow|2\rangle$ and $|2\rangle \leftrightarrow|3\rangle$ are dipoleallowed. They are characterized by the transition dipole moments $\mathrm{d}_{21}$ and $\mathrm{d}_{32}$, transition frequencies $\omega_{21}$ and $\omega_{32}$, and the spontaneous decay constants $\gamma_{21}$ and $\gamma_{32}$. The frequency $\omega_{32}$ is down-shifted with respect to $\omega_{21}$ by an amount $\Delta_{\mathrm{B}}$ being the biexciton binding energy.

The optical dynamics of an isolated SQD is governed by $3 \times 3$ density matrix. The field acting on a given SQD in the SC represents a sum of the external field and the field produced by all others SQDs in place of the given one. In this way, the total (retarded) dipole-dipole interaction is taken into account. As the mean dipole moment of an SQD depends on how strong the SQD is excited, the SQD-SQD interaction appears to depend on the current SQD's condition as well. Similar to a one-dimensional case [3], the near-zone part of the retarded SQD-SQD dipole-dipole interaction gives rise to a dynamic shift of the transition frequencies

" Corresponding author: rfmalikov@mail.ru 
$\omega_{21}$ and $\omega_{32}$, depending on the population difference of corresponding transitions. The SQDSQD interaction in the far-zone stipulates a collective radiative relaxation of SQDs, also depending on the population difference. These two effects are described by the constants $\Delta_{\mathrm{L}}$ (shift) and $\gamma_{R}$ (relaxation). Furthermore, for a SC an inequality $\Delta_{L}>>\gamma_{R}$. is always fulfilled. Just these parameters translate into a positive feedback, leading to an unusual nonlinear SC's optical dynamics.

In Fig. 1, we show the results of calculations of the field magnitude $|\mathrm{E}|$ inside the SC obtained under adiabatically sweeping the external field magnitude $\mathrm{E}_{0}$ up and down for two conditions of excitation: the external field is in resonance (i) with the one-exciton transition, $\omega_{0}=\omega_{21}$ (left panel) and (ii) with the coherent two-photon transition, $\omega_{0}=\omega_{31} / 2$ (right panel). As follows from Fig. 1, the system, similarly to the two-level model [3,4], demonstrates hysteresis: a sudden switching from one stable state to the other one, occurring at different magnitudes of the external field $\mathrm{E}_{0}$. In other words, it is bistable. Second, depending on the excitation conditions, ether the upper branch of the hysteresis loop $\left(\omega_{0}=\omega_{21}\right)$ or the lower one $\left(\omega_{0}=\omega_{31} / 2\right)$ shows windows of instability. From the analysis of the phase and Poincare maps of the field, we found that the character of these instabilities is ether of self-oscillation or chaotic nature. The inserts illustrate this.

The reflected from and transmitted through the SC fields show the identical dynamics. Furthermore, on the lower branch of hysteresis loop, the SC almost totally reflects the incident field, whereas on the upper branch, the reflection is negligible, so the system is almost transparent.

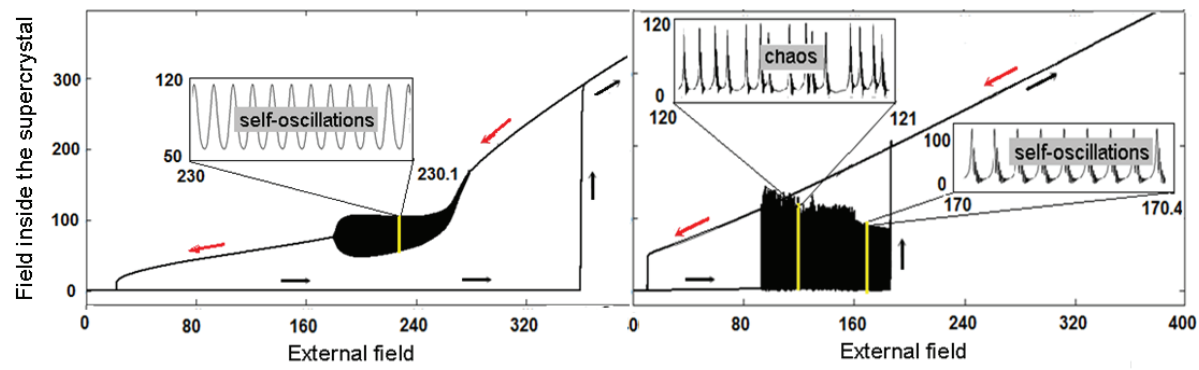

Fig. 1. The field magnitude $d_{21}|E| / \hbar$ inside the $S C$ versus the external field magnitude $d_{21} E_{0} / \hbar$, obtained under adiabatically sweeping $\mathrm{E}_{0}$ up and down (shown by arrows). Left panel $-\omega_{0}=\omega_{21}, \Delta_{\mathrm{B}}=120 \gamma_{21}$. Right panel $-\omega_{0}=\omega_{31} / 2, \Delta_{\mathrm{B}}=50 \gamma_{21}$. The rest of parameters are: $\gamma_{\mathrm{R}}=100 \gamma_{21}, \Delta_{\mathrm{L}}=1000 \gamma_{21}$. The inserts show zoomed fragments of the dynamics.

On the basis our study, we believe that supercrystals comprising SQDs might serve, first, as an all-optical switch, second, as a generator of trains of ultrashort optical pulses (in the self-oscillation regime), and, finally, as a noise generator (in the chaotic regime). These features might be of interest for all-optical technologies and nanophotonics.

R. F. M. acknowledges the M. Akmullah State Pedagogical University of Bashkortostan for a support.

\section{References}

[1] M.P. Boneschanscher, W.H. Evers, J.J. Geuchies et al., Science 344, 1377 (2014)

[2] A.S. Baimuratov, I.D. Rukhlenko, V.K. Turkov et al., Sci. Rep. 3, 1927 (2013)

[3] V. Malyshev, P. Moreno, Phys. Rev. A 53, 416 (1996)

[4] R.F. Malikov, V.A. Malyshev, Opt. Spectrosc. 122, 955 (2017) 